This paper was presented at the European Congress on Digital Pathology, 2019. The final authenticated version is available online at https://doi.org/10.1007/978-3-030-23937-4_8

\title{
Automated Segmentation of DCIS in Whole Slide Images
}

\author{
Nikhil Seth ${ }^{1,2}$, Shazia Akbar ${ }^{1,2}$, Sharon Nofech-Mozes ${ }^{2}$, Sherine Salama ${ }^{2}$ and Anne L. \\ Martel $^{1,2}$ \\ ${ }^{1}$ Department of Medical Biophysics, University of Toronto, Canada \\ ${ }^{2}$ Sunnybrook Research Institute, Toronto, Canada \\ nikhil.seth@utoronto.ca
}

\begin{abstract}
We have developed a method of segmenting DCIS lesions in WSIs using a U-Net architecture. The purpose of this study was to evaluate several different architectures and to determine the optimal resolution to field of view ratio for patches. The architecture trained at lowest resolution $(5 \mathrm{x})$ achieved the best test results $(\mathrm{DSC}=0.771, \mathrm{~F} 1=0.601)$, implying that the $\mathrm{U}-\mathrm{Net}$ benefits from having wider contextual information. A custom U-Net based architecture was trained to incorporate patches from all available resolutions. It achieved test results of DSC $=0.759, \mathrm{~F} 1=0.682$, showing improvement in the object (duct) detecting capabilities of the model. Both architectures show comparable performance to a second expert annotator on the test set. This work will be used as the preliminary part of a pipeline targeted at predicting recurrence risk in DCIS patients.
\end{abstract}

Keywords: DCIS, Segmentation, U-Net, digital pathology, deep learning

\section{Introduction}

Ductal carcinoma in situ (DCIS) is a non invasive breast cancer, accounting for approximately 2,500 new cases per year in Canada [1]. After breast conserving surgery (BCS) to remove the lesion, DCIS patients may undergo post-surgery radiotherapy to reduce the risk of developing local recurrences. Since prediction of absolute risk of recurrence based on traditional histopathologic evaluation is limited, it is not possible at present to identify patients with very low risk of recurrence in whom radiotherapy can be safely omitted. Improved stratification of patients into low- and high-risk recurrence groups would be of great benefit for a guidance-based treatment approach.

Histopathologic evaluation, of excised tissue is an important step for planning additional treatment and understanding the underlying biology of tumors which can help to some extent estimate recurrence risk. As digital slides are becoming more accessible, automation can be adopted to analyze large datasets of archived tissue. Quantitative features can be extracted from digital pathology images containing DCIS and may improve prognostication. These whole slide images (WSIs) are extremely large (several GB each) and have information stored at several different magnifications. It is impractical to extract quantitative features from an entire WSI due to computational and computer memory constraints. To efficiently extract relevant information, we need to 
effectively localize DCIS regions. Thus, there is a need for a reliable segmentation routine that can accurately identify DCIS in a WSI. This is a non-trivial problem, illustrated in Figures 1 and 3 where one can visually observe a large amount of intraclass variability for DCIS, and a comparatively small amount of interclass variability between DCIS and normal ducts. In recent years, deep learning has been shown to perform very well on detection and classification tasks in digital pathology. In this paper, therefore, we use this approach for our challenging segmentation task. Several deep convolutional neural network $(\mathrm{CNN})$ models have been proposed and evaluated. This paper will present a complete pipeline for DCIS segmentation in a WSI.

\section{$2 \quad$ Materials \& Methods}

Dataset. Our dataset consists of 202 women who were diagnosed with DCIS and underwent BCS. Specimens were handled per routine tissue processing to produce formalin-fixed paraffin embedded tissue blocks and H\&E stained sections. Representative sections were imaged in a digital slide scanner. An expert pathologist reviewed each WSI and marked annotations around the ducts that contain DCIS, using the Pathcore Sedeen ${ }^{\mathrm{TM}}$ viewer [2]. These annotations were used to create ground truth segmentations for training the CNNs. The WSI data was split into subsets for training $(\mathrm{n}=111)$, tuning $(\mathrm{n}=72)$ and testing $(\mathrm{n}=19)$. An additional $10 \%$ of the training set was held out for use as a validation set, to monitor the $\mathrm{CNN}$ for early stopping.

Training. The WSIs are far too large to be read into memory, so they must be subdivided into patches for training. Patches extracted from the set of WSIs were saved along with corresponding label images generated from the ground truth pathologist annotations. As each patch is fed through the CNN to train, it is rotated at random by either 0 , 90, 180 or 270 degrees; this augmentation increases the effective size of the training set. The CNNs are trained for 100 epochs each, and the model with the lowest validation loss from the last 10 epochs is selected as the final trained model. This training scheme allows networks sufficient training time, whilst ensuring they do not finish with a stochastically unfavourable update.

Resolution vs. Field of View. Patches can be extracted from the WSIs at different resolutions. Our images have 5x,10x and 20x resolutions available. Given a constant patch size of 256x256, patches extracted at greater resolutions will have a narrower field of view (FOV). This experiment sets out to determine which type of information the CNN finds more valuable. Patches were extracted and saved from the training set at each available resolution, creating three separate training sets. Example patches from these different resolution patch sets can be seen in Figure 1. A U-Net, like the one described in [3], was trained for each of the three resolution paradigms. Modifications to the UNet architecture include using ELU activation functions and batch normalization, as they have been shown to train networks faster [4][5]. We use padded convolutions to ensure that the output segmentation maps have the same dimensions as the input image. 


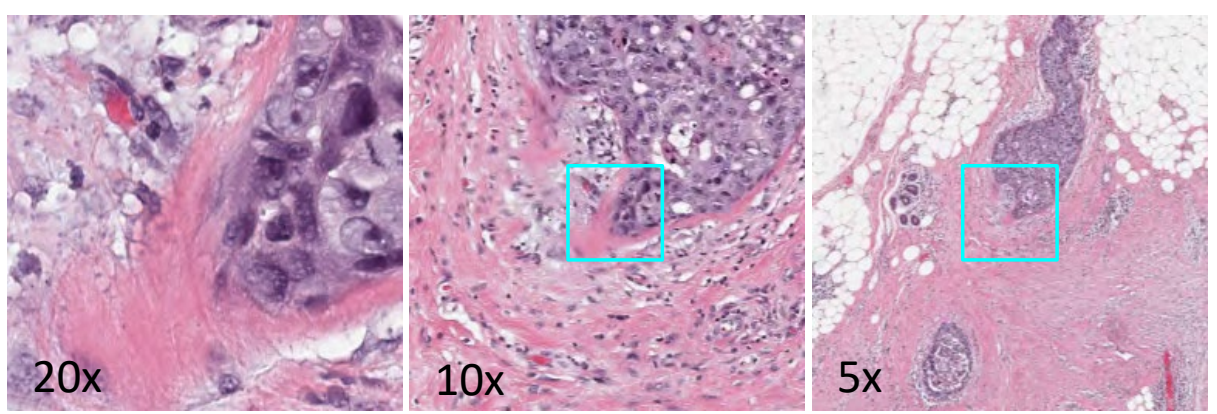

Fig. 1. Example patches from each of the three training datasets. The blue squares show the FOV of the patch one resolution step up within the context of the lower resolution patch. Patch sizes are $128 \mu \mathrm{m}(20 \mathrm{x}), 512 \mu \mathrm{m}(10 \mathrm{x}), 2048 \mu \mathrm{m}(5 \mathrm{x})$.

The mean pixelwise binary cross-entropy is calculated between the network's output patches and their corresponding label images and used as the loss function. The loss is then fed to the Adam optimizer [6] to train the network.

Multi-resolution Network. In order to overcome the resolution vs. FOV trade off, images from each resolution set were combined into a multi-resolution network. For example, all three images in Figure 1 would be treated as a single input sample, giving the network access to both high resolution details and wider contextual information. Two architectures were created to handle these inputs. One takes three patches, concatenates them into a single nine channel image, and feeds it through the same modified U-Net architecture used previously $(9 \mathrm{ch})$. The second is a custom architecture (seen in Figure 2) that splits the U-Net down-sampling arm into three convolutional branches, one for each input image, before recombining via concatenation and feeding into the up-sampling arm (3rm). The label images used to train these networks correspond to the high-resolution input images. They are also trained with the binary cross-entropy loss function and the Adam optimizer.

Evaluation. The architectures described previously are all evaluated on sets of WSIs. Each WSI has all patches extracted at the appropriate resolution. The patches are sequentially fed through the CNN being evaluated, and the outputs are stitched together to create an output probability image. This output image can be thresholded to create a binary DCIS mask for that WSI.

Dice Coefficient. The dice similarity coefficient is a measure of positive overlap between two binary images. It is defined as:

$$
D S C=\frac{2 \cdot T P}{2 \cdot T P+F P+F N}
$$

where TP is the number of true positive pixels in the images, FP is the number of false positive pixels, and $\mathrm{FN}$ is the number of false negatives. It is a similarity measure ranging from zero to one - one meaning the two images are identical. The dice coefficient 


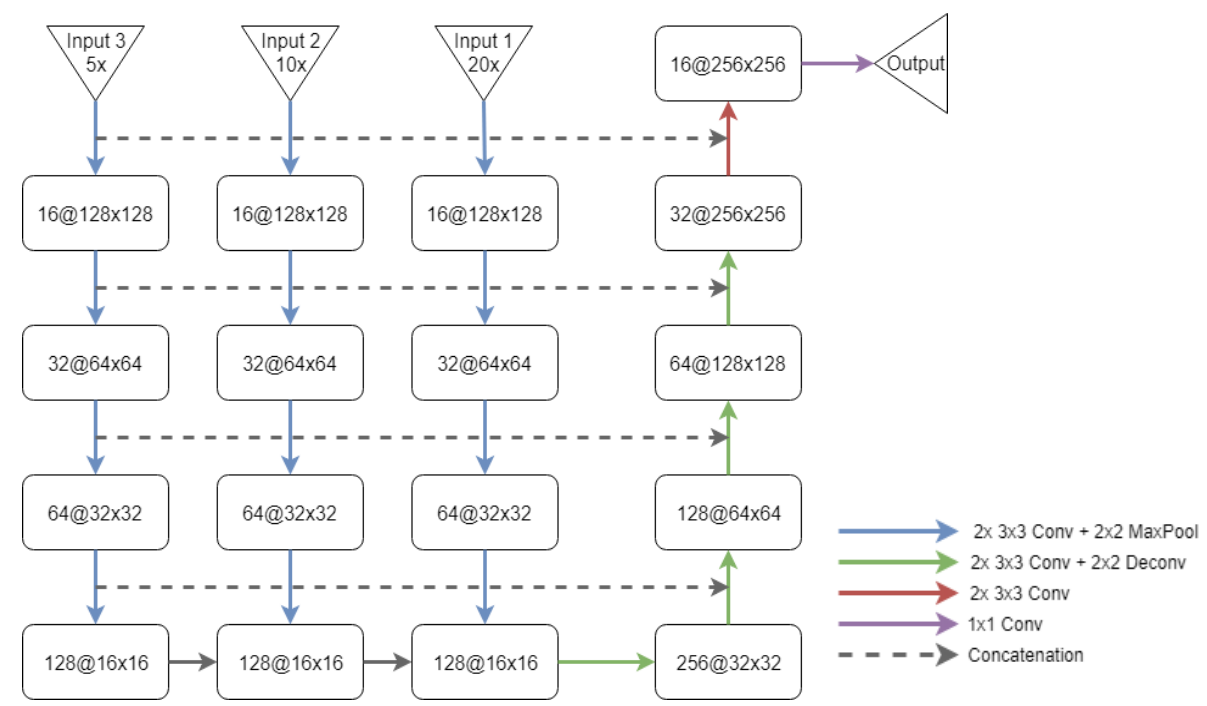

Fig. 2. Diagram of the multi-resolution U-Net (3rm). Each input is a $256 \times 256$ histology patch, and the output is a $256 \times 256$ probability map. Inputs are down-sampled separately before being combined and up-sampled.

is a useful metric for labels on a WSI because it does not depend on true negative values. This means that, unlike other metrics such as accuracy, the dice coefficient is not inflated by the high number of background pixels,.

Modified F1 Score. The F1 score is the harmonic mean of precision and recall:

$$
F_{1}=\frac{2 \cdot \text { precision } \cdot \text { recall }}{\text { precision }+ \text { recall }}
$$

The F1 score and dice coefficient are mathematically identical. However, for the F1 score, precision and recall are calculated at the object (duct) level from a binarized DCIS mask. The expert labels often group collections of ducts together as one region (figure 3), whereas the networks tend to identify each duct individually. Individually identified ducts should still be counted correctly, so precision and recall are not penalized for finding multiple predicted regions within a single ground truth region. The downside is that the F1 score is no longer symmetric; swapping the predicted and ground truth images will result in a different value for the F1 score. Henceforth, all mentions of the F1 score refer to this modified definition version of the metric.

Post-processing. A simple post-processing pipeline was created to refine the output DCIS segmentations. The pipeline consists of a thresholding operation on the probability masks, followed by a morphological opening, and finally a morphological closing. The threshold and morphological disk radius are both parameters for the post-processing pipeline, tuned sequentially using metrics calculated on the tuning set. The threshold maximizing the dice coefficient is found first. This value is then fixed when 


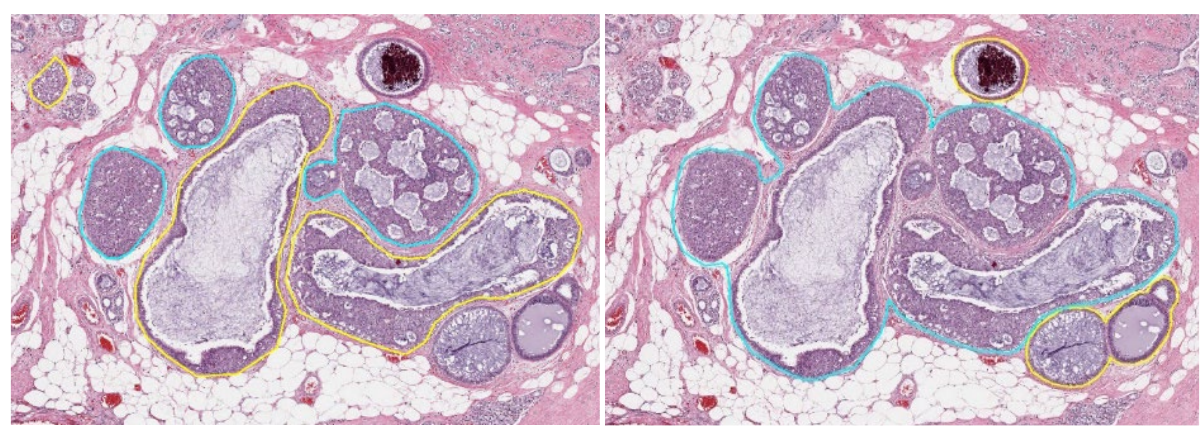

Fig. 3. Example of a group of DCIS ducts annotated independently by two different pathologists. They annotated images in two categories; definite DCIS (blue) and probable DCIS (yellow). The remainder of each image would be considered not DCIS.

tuning the morphological radius parameter. The radius maximizing the $\mathrm{F} 1$ score is taken as part of the pipeline.

Random Parameter Search. The best U-Net architecture was chosen for an extensive hyperparameter search. A random search paradigm [7] was selected, whereby the parameter space is randomly sampled and used to train the model a set number of times. This allows us to set a search budget independent of the number of parameters and possible values. The efficiency of the search also does not decrease by adding extra parameters that do not affect the performance. Preliminary tests were performed to identify valid ranges for each parameter being included. The search ran 100 times, each with a random set of hyperparameters sampled from their ranges. Each model was trained with a 5 -fold cross-validation.

Annotator Similarity. In order to gauge the effectiveness of these automated systems, it is useful to compare to human level performance. Two expert pathologists were asked to independently annotate all the DCIS on the held-out test set of 19 WSIs. The dice coefficient and F1 score were measured comparing the pathologists' performance relative to each other. Figure 3 shows an example of such paired independent annotations. This image illustrates the significant variability between observers. These annotator similarity comparisons serve as a benchmark for the described automated techniques.

\section{$3 \quad$ Results \& Discussion}

Resolution Architecture Evaluation. Test results for each resolution architecture, including their post-processing pipelines, can be seen in Table 1. Each network was run on the independent test set used to compare annotators and evaluated against the annotator who created the original training labels. The $5 \mathrm{x}$ model achieves the greatest dice coefficient and F1 score of the single resolution networks. This implies that the segmentation quality benefits from patches with a greater FOV, as the network can make 


\begin{tabular}{l|l|l|l|l|l}
\hline Model & Dice Score & Dice P-Value & F1 Score & F1 P-Value & Time (s) \\
\hline $\begin{array}{l}\text { Second } \\
\text { Annotator }\end{array}$ & 0.732 & N/A & 0.427 & N/A & N/A \\
\hline $5 \mathrm{x}$ & 0.771 & 0.314 & 0.601 & 0.077 & 6.35 \\
\hline $10 \mathrm{x}$ & 0.558 & $0.009^{*}$ & 0.478 & 0.573 & 93.16 \\
\hline $20 \mathrm{x}$ & 0.617 & 0.184 & 0.546 & 0.147 & 2247.89 \\
\hline $3 \mathrm{rm}$ & 0.759 & 0.398 & 0.682 & $0.006^{*}$ & 3065.07 \\
\hline $9 \mathrm{ch}$ & 0.691 & 0.546 & 0.662 & $0.016^{*}$ & 3177.02 \\
\hline
\end{tabular}

Table 1. Results for each architecture run on the test set. P-values are from Wilcoxon sign-rank tests comparing each model to the second annotator. Asterisks indicate statistical significance.

use of the wider contextual information. This makes sense considering that pathologists mostly determine which regions contain DCIS from observing the WSI at low resolution, using higher resolutions to fine tune their decisions. The $5 \mathrm{x}$ network also benefits from training and running in drastically less time than other comparable quality architectures, due to the down-sampled nature of WSIs being processed. The 3rm multiresolution architecture has the best F1 score of all the networks, and a dice coefficient comparable to the $5 \mathrm{x}$ network. Thus, the $3 \mathrm{rm}$ network was successfully able to combine information from high and low resolutions to increase the segmentation accuracy and duct detecting capabilities of the model, however this was at the cost of significantly longer processing times. An ROC analysis, as seen in Figure 5, further supports the conclusion of $5 \mathrm{x}$ being the best model. Its curve has an AUC of 0.987 and is the closest to the top left across the entire curve. Interestingly the $3 \mathrm{rm}$ shows slightly worse performance than the $5 \mathrm{x}$ model, though it is still an improvement over all the other models.

Comparison with Second Annotator. A Wilcoxon sign-rank test was performed to compare each models' results to the annotator similarity metrics. When comparing dice coefficients, only the $10 \mathrm{x}$ network was found to be significantly different (worse) than the second annotator. No other networks show statistical differences from the second annotator, implying they have comparable performance. When comparing the F1 scores, both multi-resolution models showed statistically significant improvement over the second annotator. It is important to note the small sample size of dually annotated WSIs in this test set. More labelled test data would be helpful in teasing out the performance differences between each model.

Random Search Results. The 5x based U-Net model was chosen for the random parameter search, due to its superior performance and drastically reduced run time. The randomly generated models were each run and evaluated on the tuning set. The area under the receiver operating characteristic (AUC) was calculated and averaged across all folds for each model. A series of iterative Kruskal-Wallis tests was performed on the model set. At each iteration, the test is performed. If it is significant $(p<0.05)$, the lowest mean AUC model is removed from the set. This process is repeated until the test is not significant, resulting in a set of the top models that are not significantly different from one another. The results of this analysis can be seen in Figure 5. The set of top 
(a)

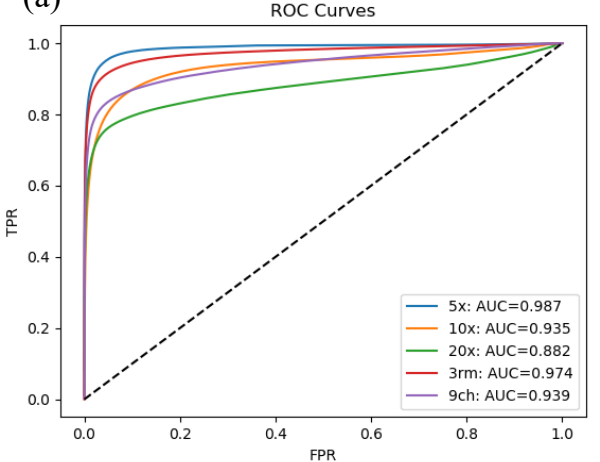

(b)

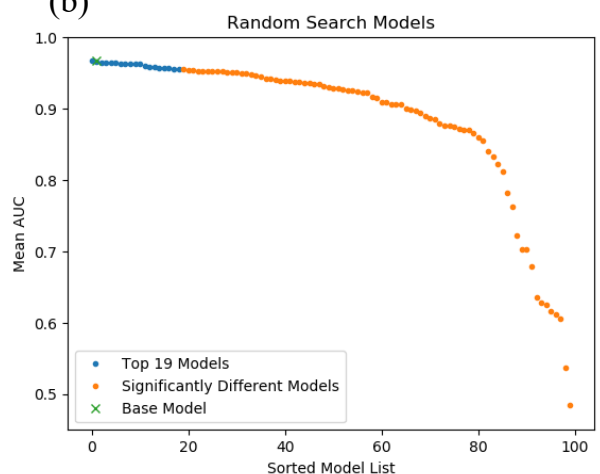

Fig. 4 (a). ROC curves for each model, run on the testing set. The ROC analysis requires the raw probability values, and thus does not include the post-processing pipeline for each model.. (b). Models resulting from the random parameter search, sorted by mean test AUC. Blue represents top set of models showing no difference from the Kruskal-Wallis test. Orange is all other models. The green $\mathrm{x}$ represents the model with the baseline hyperparameter set.

models includes the base model with original hyperparameters. Most models produced by the search did not improve on the base model, but a reasonably large group $(n=19)$ are shown to have not significantly different performance from the base model. This implies that the U-Net architecture functions quite well for this problem as designed, and small hyperparameter alterations can have minimal effects on the performance. Thus, the baseline hyperparameters were chosen to move forward.

Sample Output. Figure 5 shows an example of the 5x model being run on a WSI from the test set, compared to annotations from one of the pathologists. The predicted segmentation regions are generally very close to the annotation borders, with large drops in probability marking the edges of predicted DCIS regions. Some additional ducts are detected, albeit with low probability. This is acceptable because the next task will involve extracting features from the DCIS duct boundaries, meaning it is more important that the model finds correct and well-defined edges of the DCIS regions. This is achieved by optimizing the algorithm to have the best dice coefficient. Optimizing for the best F1 score would yield an algorithm suited to correctly identifying all DCIS ducts, whilst allowing for errors on the boundaries. With more refinement, this could be implemented as a processing step on slide scanners to identify DCIS regions immediately as slides are processed, aiding pathologists in their analysis.

\section{Conclusion}

This paper presented an analysis of using established and novel CNN architectures to solve the problem of localizing DCIS on a WSI. It was found that when using a traditional U-Net architecture, there are both speed and accuracy benefits to training and running the model using low resolution patches. This is due to the patches having a greater FOV, giving the U-Net access to wider contextual information. Two novel 


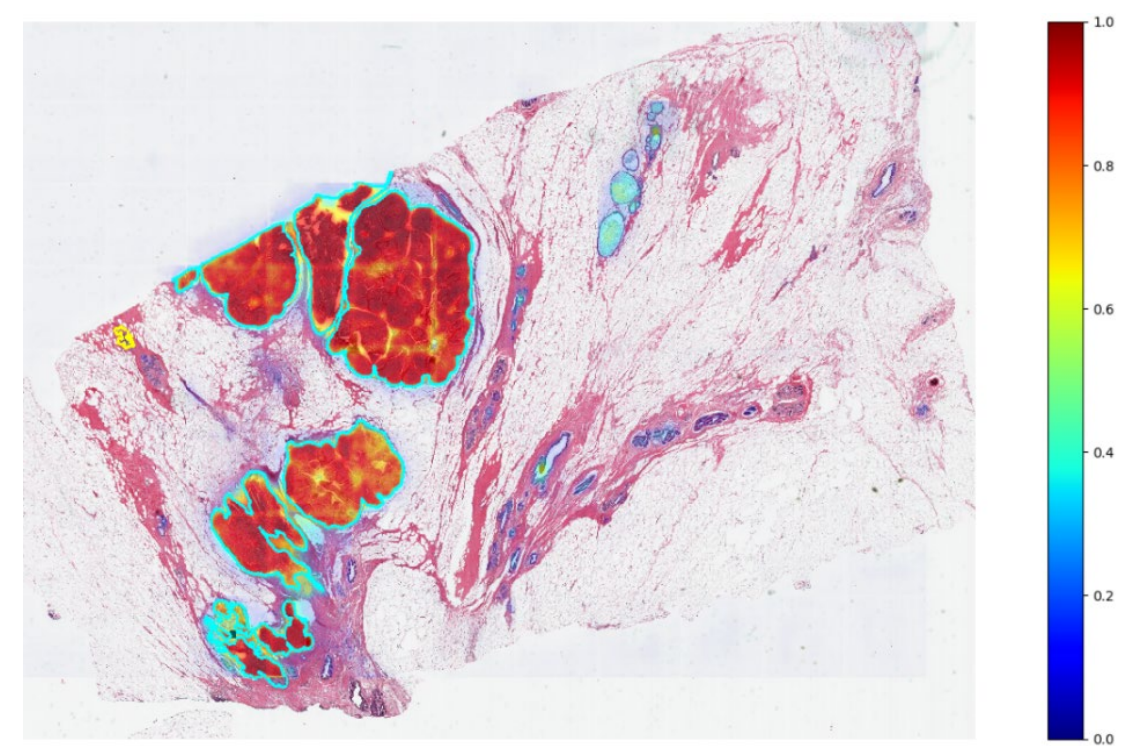

Fig. 5. Example WSI from the test set. Blue outlines indicate a pathologist labelled DCIS region. The colour overlay represents the probability map output by the $5 \mathrm{x}$ based model. Red indicates high probability and blue is low. Zero values are set to be transparent.

multi-resolution architectures were presented, that combined patches from all three available resolutions to overcome the resolution vs. FOV trade-off. However, this comes at the expense of training and running times for the networks. Finally, a comparison was made between two independent pathologist annotators, showing that the best networks do approximately as well as the second annotator on the segmentation task. The next steps for this project involve performing feature extraction on DCIS regions identified by the networks and beginning to build new models that can predict recurrence risk for DCIS patients.

\section{Acknowledgements}

This work was funded by the Canadian Cancer Society Grant \#705772, and the Canadian Breast Cancer Foundation.

\section{References}

1. Canadian Cancer Statistics.

2. A. L. Martel et al., "An Image Analysis Resource for Cancer Research: PIIP-Pathology Image Informatics Platform for Visualization, Analysis, and Management," Cancer Res., vol. 77, no. 21, pp. e83-e86, Nov. 2017. 
3. O. Ronneberger, P. Fischer, and T. Brox, "U-Net: Convolutional Networks for Biomedical Image Segmentation,” Med. Image Comput. Comput. Interv. -- MICCAI 2015, vol. 9351, pp. 234-241, May 2015..

4. D.-A. Clevert, T. Unterthiner, and S. Hochreiter, "Fast and Accurate Deep Network Learning by Exponential Linear Units (ELUs)," In: ICLR (2016)

5. S. Ioffe and C. Szegedy, "Batch Normalization: Accelerating Deep Network Training by Reducing Internal Covariate Shift," In: ICML 2015.

6. D. P. Kingma and J. Ba, “Adam: A Method for Stochastic Optimization,” In ICLR 2015.

7. J. Bergstra, J. B. Ca, and Y. B. Ca, "Random Search for Hyper-Parameter Optimization Yoshua Bengio," J. Mach. Learn. Res. 13, 281-305 (2012). 